\title{
LAS PALMERAS NATIVAS DE IMPORTANCIA ECONOMICA EN LA AMAZONIA PERUANA.
}

\author{
(*) KAHN Francis \\ $(* *) \quad$ MEJIA Kember
}

\section{RESUMEN}

La mayoría de las palmeras son utilizadas por el selvático amazónico obteni6ndose de ellas una variedad de productos, como frutas, aceite, palmito, materiales de construcción y cestería. Sin embargo, son pocas las especies que tienen un buen potencial económico. Estas se encuentran principalmente en suelos hidromórficos inundados, que cubren gran parte de la amazonía peruana. Tres especies promisorias - Jessenia bataua, Mauritia flexuosa y Euterpe precatoria forman poblaciones muy densas en estos suelos, generalmente considerados como impropios para la agricultura. El manejo racional de las poblaciones de palmeras promisorias contribuirá a mejorar la potencialidad económica de estos suelos, al transformar las formaciones vegetales en agroselvas de producción sostenida.

\section{SUM MARY}

M ost palm species are used by A mazonian natives and provide a variety of products, such as edible fruits, oil, palm heart, building materials, and basketry. $\mathrm{Ho}$ wever, only a few species have significant economic potential. These occur essentially on waterlogged or flooded soils which cover vast areas in Peruvian A mazonia. Three especially promising species Jessenia bataua, Mauritia fleuosa sud Euterpe precatoria. Constituite dense populations on these sois, which are generally considered as unfit for agriculture. The management of promising palm populations will contribute to increasing the economic value of such soils by transforming seasonal swamp forests into productive agroforestry fields.

\section{(*) Responsable ORSTOM}

$(* *)$ J efe de Proyecto C entro de Investigaciones de J enaro Herrera 


\section{INTRODUCCION}

Los primeros exploradores de la amazonía, botánicos y zoólogos, fueron particularmente atraídos por las palmeras. A. R. WALLACE le consagró un libro, "Palm trees of the A mazon and their uses", publicado en 1853. y los nombres de BARBOSA RODRIGUES, D'ORBIGNY, MART][US, PAVON, RUIZ, SPRUCE, TRAIL están definitivamente ligados a las palmeras de la cuenca amazónica.

Todas las partes de la planta se aprovechan: hojas (techado, paredes, cestería, fibras); pecíolo (piezas de construcción, cestería, esteras, flechas); tronco (pare" des, pisos, puertas, almidón, cerbatanas); palmito; frutos (alimento, aceite, carbón) y algunas raíces para medicina tradicional (ANDERSON 1978, BALICK 1979, 1985, BOOM 1986, BRAUN 1968, CAVALCANTE 1974, KAHN Y MEJIA 1987, LEVI. STRAUSS 1950, MEJIA 1903, 1987, SCHULTES 1974, 1977, WALLACE 1853).

A unque hay múltiples usos para las palmeras, son pocas las especies que tienen una significación económica potencial: sin embargo, hay especies con alta producción de frutos comestibles, ricos en aceite, así como palmitos aceptables para la industria conservera o para la producción de almidón, etc. Tales plantas no serán definidas aquí en función de sus rendimientos agroeconómicos, dado que son plantas nativas que necesitan un programa de mejoramiento genético antes de que sean competitivas con las plantas cultivadas ya mejoradas.

No se trata aquí de proponer plantaciones intensivas de palmeras nativas, como las de palmera aceitera africana o de cocotero, cuyas productividades actuales son el resultado de más de cincuenta años de mejora genética, sino de proponer una explotación racional de las poblaciones de palmeras nativas en la amazonía.

En esta óptica, los suelos sobre los cuales crecen las palmeras nativas constituirán el criterio de mayor importancia para definir las especies con potencial económico: sobre suelos buenos, las plantas cultivadas tradicionalmente of recen una producción mucho más rentable que las palmeras nativas; pero, sobre suelos no aptos para la agricultura, esta producción es muy baja. Por ese motivo, el manejo racional de palmeras nativas constituye una de las mejores alternativas que se tiene para el mejor aprovechamiento de estos suelos.

A unque no es el objetivo inmediato, especies relacionadas con las palmeras de reconocida importancia económica, serán también consideradas como fuente potencial de germoplasma para el futuro mejoramiento genético de dichas especie. 


\section{PALMERAS DE IMPORTANCIA ECONOMICA EN LA AMAZONIA PERUANA}

Se ha considerado ocho especies con potencial económico (KAHN 1987), las cuales de acuerdo a sus posibilidades de uso son las siguientes:

\section{Aceite de palmeras}

J essenia bataua ( $M$ art.) Burret (ungurahui) es una palmera arborescente, hasta $25 \mathrm{~m}$. de alto, monocaule. El aceite de .J. bataua es muy similar al de oliva (BALICK 1981, 1982) y la pulpa contiene proteínas de alta calidad (BALICK y GERS. HOFF 1981). Los nativos usan el aceite de J. bataua para cocinar, para el pelo y para lubricar sus armas; con la pulpa, preparan una bebida muy apreciada (SCHWYZER 1981). Las hojas se utilizan para el techado de construcciones provisionales y la confección de cestas (capillejos); los troncos se utilizan eventualmente como horcones en la construcción de viviendas.

Oenocarpus mapora Karst., 0 . Lacaba Mart. (Sinamillo), son palmeras multi y monocaule respectivamente, de 10 a $15 \mathrm{~m}$. de alto. Estas especies forman parte de un complejo genético que incluye .J . bataua (BALICK 1981, 1986, FORERO 1983, MARTIN y GUICHARD 1979). Los frutos son utilizados ocasionalmente para preparar una bebida; con la corteza del raquis de las hojas se confecionan cernidores y cestos, los troncos pueden ser utilizados corno horcones en las casas, también se utilizan para el techado de las viviendas. A simismo, constituye una especie promisoria para el establecimiento de un banco de genes del complejo J essenia 0 enocarpus

Elaeis oleifera (H.B.K.) Cortés (poloponta) es una palmera de tronco rastrero. Poblaciones de esta especie están siendo buscadas en diversas partes de la amazonia para su mejoramiento e hibridación con la palmera aceitera africana, Elaeis guineensis Jacq. La especie de la amazonía ofrece un aceite de alta calidad, una escasa altura debido a su tronco reptante, que facilita la cosecha de los racimos y una mejor resistencia a diversas enfermedades (podredumbre del cogollo, manchas anulares y marchitez sorpresiva) que atacan gravemente la palmera africana cultivada en la cuenca amazónica (MEUNIER 1975, 001 et al. 1981). En el Perú, curiosamente, esta especie no tiene ningún uso tradicional, reportado a la fecha.

\section{Frutos comestibles y almidón}

Astrocaryuzn chambira Burret (chambira), es una palmera arborescente, hasta de $25 \mathrm{~m}$. de alto. El endosperma inmaturo, semejante al de coco, se bebe y se come. 


\section{6}

Be las hojas se hacen sombreros y abanicos y de la epidermis de los foliolos de la hoja más joven se obtienen fibras muy resistentes y apreciadas para la confección de hamacas y bolsas.

Bactris gasipaes H.B.K. (pijuayo), es una palmera multicaule, de 10-20 m. de al to. Se cultiva en toda la cuenca amazónica por sus frutos (alimento y bebida), no ha sido encontrado en poblaciones naturales. El tronco se utiliza en la construcción de paredes, de arcos y puntas de flechas, principalmente de artesanía.

M auritia flexuosa L.f. (aguaje), es una palmera monocaule, arborescente, que puede alcanzar más de $30 \mathrm{~m}$. de alto. Es la palmera más frecuente en la amazonía peruana. Sus frutos y derivados (refrescos y helados) se venden en las calles de Iquitos y demás ciudades, constituyendo localmente un producto comercial de cierta importancia (PADOCH 1987). Esta especie es dioica.

Actualmente, las plantas masculinas son usadas como un recurso para la obtención de larvas comestibles de coleópteros del género Rhynchophorus, "suris". Sin embargo, el tronco de M. flexuosa contiene altas concentraciones de almidón; este producto permitiría el uso económico de las plantas masculinas, convirtiendo esta especie en una fuente de almidón para toda la región amazónica (RUDDLE et al. 1978). En las zonas rurales se confeccionan esteras con la corteza del pecíolo de las hojas y, con la médula, tapones (corchos) para las botellas.

\section{Palmito}

Euterpe precatoria $M$ art. (huasai), es una palmera monocaule, arborescente, hasta de $20 \mathrm{~m}$. de alto, tradicionalmente utilizada para la obtención de palmito en la amazonía peruana; esta especie es menos apropiada para la industria conservera que las especies multicaules, como $\mathrm{E}$. oleracea $\mathrm{M}$ art. 0 Bactris gasipaes. Sin embargo una fábrica en Iquitos la está utilizando, lo que obliga ir a localidades muy alejadas de la ciudad para encontrar poblaciones extensas de esta palmera. En las zonas rurales y áreas marginales de las ciudades, se utiliza el tronco de E. precatoria para la construcción de paredes de las viviendas (LOPEZ PARODI 1987).

\section{ECOLOGIA DE LAS PALMERAS DE IMPORTANCIA ECONOMICA}

L as palmeras de importancia económica anteriormente descritas se encuentran en seis ecosistemas forestales, bien caracterizados por la vegetación y el suelo (Clasificación FA O-U NESCO 1971): bosque de altura sobre acrisol o luvisol, bosque de quebrada sobre gleysol, aguajal sobre histosol, bosque de restinga sobre fluvisoL, chamizal sobre podzol órtico, ungurahual sobre podzol gleyco (Tabla 1). 
Estos ecosistemas están bien representados en la amazonía baja, incluyendo el piedemonte andino oriental hasta los $700 \mathrm{~m}$. de altitud. No fueron encontradas palmeres de importancia económica en los bosques de tahuampa, inundados por aguas negras.

\section{Bosque de altura sobre acrisol o luvisol}

Las especies de importancia económica son poco frecuentes en los bosques de altura sobre acrisol o luvisol, que son suelos generalmente bien drenados. Sin embargo, es precisamente en estos bosques de altura en que se encuentran la riqueza específica mas alta en palmeras. Por ejemplo, en el bajo U cayali se han encontrado 29 especies en $0.71 \mathrm{Ha}$. (KAHN et al. 1988). Solamente cuatro de estas son especies promisorias: Astrocaryum chambira, O enocarpus bacaba, J essenia bataua y Euterpe precatoria. Las dos primeras se encuentran en estado adulto, pero siempre con una densidad muy baja (1-5 palmeras adultas por $\mathrm{Ha}$.); las demás están únicamente representadas por plántulas y juveniles. Poblaciones densas de A strocaryum chambira se encuentran en vegetaciones secundarias (purmas), pastizales, y cerca de los pueblos, cuando es propagada por el hombre.

\section{Bosque de quebrada sobre gleysol}

Estos bosques se encuentran en las terrazas bajas, solamente inundables por las fuertes Iluvias que caen en la estación lluviosa. El suelo se caracteriza por presentar un gley, es decir un horizonte hidromórfico con olor a anhídrido sulfuroso (H2S), y por una acumulación de materia orgánica cerca de la superficie. Se encuentran cuatro especies con frecuencias altas y densidades medias a altas: I. bataua, M. flexuosa, E. precatoria y 0 . mapora (Tabla II). En $1 \mathrm{Ha}$. de esta formación, estudiada en el bajo U cayali, estas cuatro especies promisorias constituyen hasta $36.3 \%$ del área basal de la parcela. La palmera aceitera americana, E. oleifera, ocurre con baja frecuencia en lugares semejantes, pero puede formar poblaciones muy densas (KAHN y MEJIA 1986).

\section{Aguajal sobre histosol}

El nombre de esta vegetación se debe al aguaje, M. flexuosa. Tales formaciones, mencionadas por SPRUCE (1871), BOUILLENNE (1930) y MOORE (1973), cubren vastas áreas de la amazonía, desde el piedemonte oriental de los A ndes hasta la costa A tlántica; siempre sobre suelos anegados. En la Guyana Francesa, las formaciones de $M$. flexuosa se encuentra casi exclusivamente en la sabana costera (GRANVILLE 1978). 
Estas formaciones son particularmente extensas en la amazonía peruana. Un análisis de imágenes LANDSAT (ONERN 1977), muestra que las mismas cubren el $21 \%$ de $311,970 \mathrm{Ha}$. cerca de Iquitos y el $34 \%$ de $66,560 \mathrm{Ha}$. en el valle del río M arañon.

M. flexuosa alcanza densidades muy altas en poblaciones naturales un promedio de 246 palmeras con tronco por Ha. (CV : 24.7\%) fue obtenido a través do un inventario realizado por SALAZAR y ROESSL (1977), a partir de 10 parcelas de $0.5 \mathrm{Ha}$. cada una localizadas a lo largo del río I taya, cerca de Iquitos. En el valle del A Ito Huallaga GONZALES (1971) encontró un promedio de 351 palmeras con tronco (CV:26\%) y 297 juveniles (CV: 43\%) por $\mathrm{Ha}$, a partir de 20 parcelas de $0.5 \mathrm{Ha}$. cada una.

En el bajo U cayali se encuentran formaciones semejantes, situadas en terrazas bajas. El suelo es un histosol dístrico, compuesto por materia orgánica; constituida principalmente por deshechos de M. flexuosa: hojas caídas, inflorescencias masculinas e infrutescencias.

El área basal total (para DAP mayor a $15 \mathrm{~cm}$ ) es alta, con $31.11 \mathrm{~m}^{2} / \mathrm{Ha}$., de estos $53.1 \%$ corresponden a $M$. flexuosa, con 230 palmeras con tronco, entre ellas 138 adultos. Estas formaciones incluyen $\mathrm{E}$. precatoria y 0 . mapora (Tabla II). Jessenia bataua no se encuentra en estas formaciones.

\section{Bosque de restinga sobre fluvisol}

Estos bosques inundables durante varios meses al año, corresponden al concepto brasileño de "várzea" (PRA NCE, 1980).

El suelo es una acumulación de sedimentos depositados durante la creciente de los ríos. Dos especies promisorias se encuentran con alta frecuencia y densidad media: E. precatoria y 0 . mapora.

\section{Chamizal sobre podzol órtico}

El chamizal es una vegetación muy baja, seca, que corresponde al concepto brasileño de "campinas" (ANDERSON 1981, GUILLA UMET 1987). EI suelo se caracteriza por un horizonte de arena blanca sobre una capa consolidada de hierro y materia orgánica muy impermeable. Después de las lluvias, el agua detenida en profundidad por esta capa se evacúa lateralmente en el horizonte superior de arena 
blanca. Resulta de ello un suelo muy seco. Las palmeras de importancia económica están representadas solamente por algunos individuos de J essenia batua.

\section{Ungurahual sobre podzol gleyco}

El nombre de estos bosques se debe al ungurahui, J. bataua, que forma poblaciones muy densas (Tabla II). El suelo tiene una estructura muy semejante a la del podzol, se distingue por su hidromorfismo; consecuencia de su posición topográfica en una depresión que favorece la permanencia del agua en el horizonte superior de arena blanca, al ser retenida por la capa consolidada en profundidad y no poder drenar lateralmente.

\section{LAS PALMERAS DE IMPORTANCIA ECONOMICA Y EL MANEJO DE LOS BOSQUES}

A través de la información presentada se aprecia claramente que varias de las especies de palmeras promisorias de la amazonía peruana forman poblaciones densas y extensas en suelos anegados, histosol y fluvisol o de mal drenaje, gleysol y podzol gleyco, siendo éste último, además, muy pobre en nutrientes (Tabla 1).

Estos suelos, que no son aptos para la agricultura, cubren vastas áreas, constituyendo una seria limitación para el desarrollo económico de la región. El manejo racional de las poblaciones naturales de palmeras nativas puede permitir el uso de tales suelos e integrarse como parte importante de un programa regional de ecodesarrollo. El objetivo es transformar los bosques de suelos hidromórficos o inundados en campos agroforestales productivos que incrementen la capacidad económica de los pobladores de la región. Las tres especies con altas densidades en la amazonía peruana J . bataua, M. flexuosa y E. precatoria, pueden ser utilizadas integralmente, ya que además de su valor económico potencial, son utilizadas también como recursos para materiales de construcción, fibras, etc.

En todos los países tropicales, las palmeras de suelos hidromórficos son comúnmente utilizadas. En la amazonía brasileña, ANDERSON et al. (en prensa) describen la integración de Euterpe oleracea en un sistema agroforestal. Ejemplos de A sia y de las islas del Océano Pacífico son descritos por RUDDLE et al. (1978) y BARRAU (1959), respectivamente. Los usos de Raphia en África son listados por PROVFIZI (1983), quien propone un sistema de manejo racional de estos pantanos, basado en las palmeras. 
Sin embargo, se considera que dos puntos preliminares deben ser resueltos para que un plan de manejo de las poblaciones naturales de palmeras nativas en la amazonía peruana pueda tener éxito.

En primer lugar, un manejo racional de tales poblaciones naturales debe iniciarse con un cambio radical de las prácticas de cosecha de los frutos. A ctualmente los frutos son colectados cortando las palmeras, lo que trae como consecuencia que la vegetación nativa pierda progresivamente su potencial económico, En el caso de la especie dioica Mauritia flexuosa, es corriente una preponderancia de plantas masculinas entre las palmeras adultas, reflejando las prácticas de cosecha pasadas (tabla III). Este problema de manejo también existe en otros países amazónicos. GRANVILLE (1985), en la Guyana Francesa, ha iniciado una campaña para evitar que se corten las palmeras para cosechar los frutos.

En segundo lugar, es necesario introducir nuevos usos para las palmeras. EI almidón contenido en el tronco del aguaje, M. flexuosa, sólo ha sido utilizado de manera tradicional por los grupos nativos WARAO del delta del Orinoco en la amazonía venezolana (HEINEN y RUDDLE 1974). Sin embargo, varias especies de palmeras, principalmente de los géneros A renga y $M$ etroxylon, son explotadas como fuentes de almidón en el sureste de Asia y, en varios países, la comercialización del almidón de palmera constituye una actividad económica importante a nivel regional, corno lo muestra el desarrollo de manufacturas modernas para la extracción de este producto (RUDDLE et al. 1978). Una transferencia de la tecnología de A sia permitiría la explotación del almidón del aguaje y la valorización de las poblaciones naturales de dicha palmera, utilizando también las plantas masculinas.

La parte peruana de la cuenca amazónica se caracteriza por las extensas áreas de inundación temporal o permanente y de suelos hidromórficos, en relación con el área total. Tales áreas están cubiertas por ecosistemas forestales poco diversificados, esencialmente compuestos por palmeras de importancia económica potencial. Estos ecosistemas, instalados sobre suelos no aptos para la agricultura, están poco destruidos, En cambio, los bosques de altura, caracterizados por una diversidad muy grande, son cortados de modo incontrolable. En algunas regiones, la situación es catastrófica, como es el caso del Departamento de San Martín, en el cual casi todos los bosques de altura fueron destruidos, salvo en al gunas áreas muy reducidas.

Es de suma urgencia que aprendamos a manejar las poblaciones naturales, como las palmeras nativas y evitar talar nuevos bosques, conservando así las riquezas naturales que las generaciones futuras podrían no conocer nunca, si se sigue explotando los bosques húmedos tropicales de modo totalmente irracional. 
TABLA 1 PALMERAS DE IMPORTANCIA ECONOMICA EN LOS ECOSISTEMAS FORESTALES DE LA AMAZONIA PERUANA (*)

$\begin{array}{lll}\mathrm{Fa} & : & \text { Frecuencias de Palmeras adultas } \\ \mathrm{O} & : & \text { Nunca o excepcional } \\ 1 & : & \text { Escasa } \\ 2 & : & \text { Media } \\ 3 & : & \text { Alta } \\ 4 & : & \text { Siempre } \\ \mathrm{Da} & : & \text { Densidad adultas } \\ 1 & : & \text { Baja } \\ 2 & : & \text { Media } \\ 3 & : & \text { Alta } \\ 4 & : & \text { Siempre }\end{array}$

Bosque de Bosque de Bosque de Chamisal Ungurahual Altura sobre quebrada A guajal restinga sobre sobre acrisol o sobre sobre sobre podzol podzol luvisol cleysol histosol fluvisol ortico gleyco

\section{Fa Da Fa Da Fa Da Fa Da Fa Da Fa Da}

$\begin{array}{llllllllllllll}\text { A strocaryum chambira } & 2 & 1 & 0 & - & 0 & - & 0 & - & 0 & - & 0 & - & \\ \text { Elaeis oleifera } & 0 & - & 1 & 3 & 0 & - & 0 & - & 0 & - & 0 & - & \\ \text { Euterpe precatoria } & & 0 & - & 4 & 3 & 42 & 3 & 2 & 2 & 0 & - & 1 & 2 \\ \text { Jessenia bataua } & 0 & - & 4 & 3 & 0 & - & 0 & - & 2 & 1 & 4 & 3 & \\ \text { M auritia flexuosa } & 0 & - & 4 & 3 & 4 & 3 & 0 & - & 0 & - & 0 & - & \\ \text { Oenocarpus bacaba } & 2 & 2 & 0 & - & 0 & - & 0 & - & 0 & - & 0 & - & \\ \text { Oenocarpus mapora } & 0 & - & 4 & 23 & 4 & 23 & 2 & 2 & 0 & - & - & - & \end{array}$

(*) Bactris gasipaes especie cultivada no encontrada en poblaciones naturales en la amazonía peruana 
112

\section{TABLA 2 ECOSISTEMAS MAS RICOS EN PALMERAS DE IMPORTANCIA ECONOMICA:}

Densidades por $1 \mathrm{Ha}$. En poblaciones situadas en el bajo U cayali en zona de Jenaro Herrera.

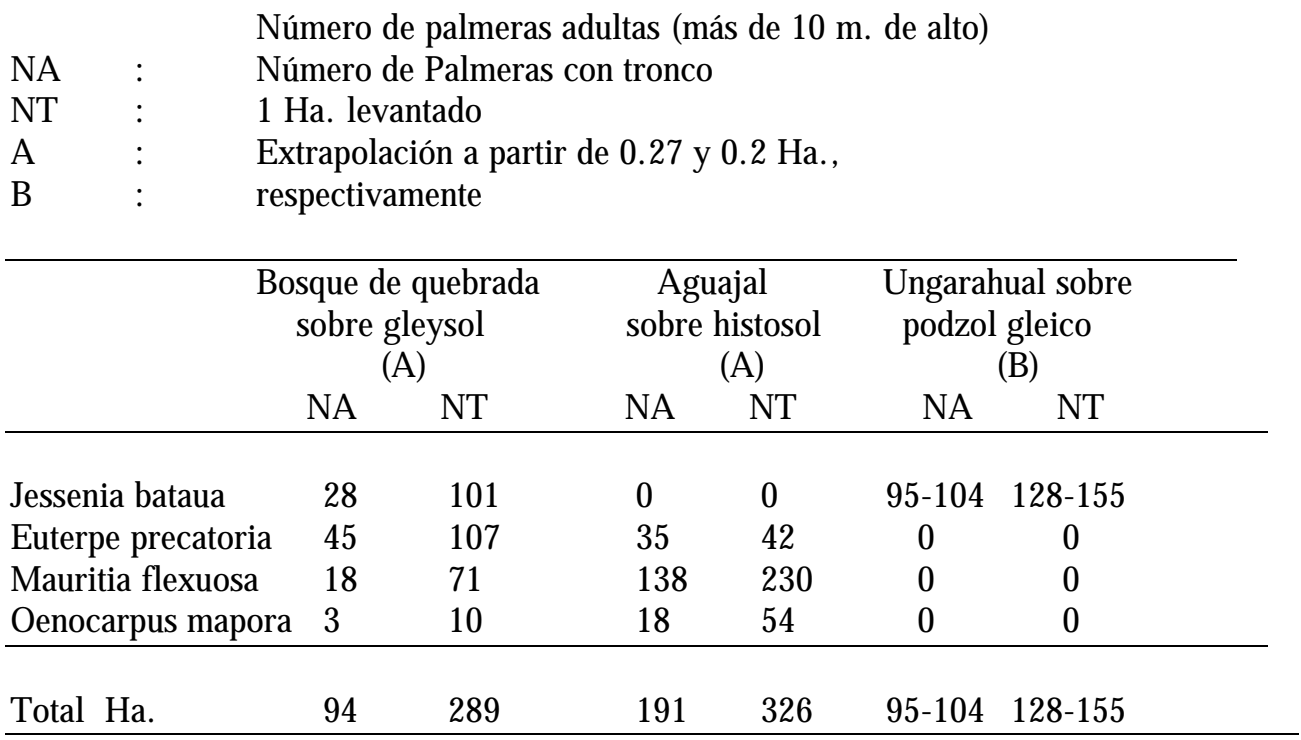

TABLA 3 SEX-RATIO EN CUATRO POBLACIONES DE Mauritia flexuosa

$\begin{array}{lllc}\text { Bosque de } & \text { A guajal } & \text { A guajal } & \text { A guajal } \\ \text { quebrada } & 1 \mathrm{Ha} .(\%) & 0.5 \mathrm{Ha} .\left(^{*}\right) & 1 . \mathrm{Ha} .(007) \\ 1 \mathrm{Ha} .\left(^{*}\right) & & & \end{array}$

Árboles masculinos $15(83.33 \%) \quad 100(72.469 \%) \quad 13(68.42 \%) \quad 11(61.6 \%)$ Árboles femeninos $3(16.66 \%) \quad 23(16.67 \%) \quad 4(21.05 \%) \quad 69(38.3 \%)$ Sexo no identificado $0 \quad 15(10.86 \%) \quad 2(10.86 \%) \quad$ ?

\begin{tabular}{lllll} 
Total & 18 & 138 & 19 & 180 \\
\hline
\end{tabular}

* En el bajo U cayali zona de Jenaro Herrera

* En el Itaya (Salazar y ROESSL, 1977) 


\section{REFERENCIAS BIBLIOGRAFICAS}

ANDERSON A. B. 1987, The names and uses of palms among a tribe of $Y$ anomana Indians. Principes 22 (1): 30-41

ANDERSON A.B. 1981 . White sand vegetation of Brazilian A mazonia. Biotropica 113 (3): 199210.

ANDERSON A. B, A.. GELY, J, STRUDWICK, G. L. SOBEL, \& M. G. C. PINTO . In press. Um sistema agroforestal na várzea do estuario amazónico ( ilha das oncas, M unicipio de Barcarena. Estado do Pará). A cta A mazónica.

BALICK M. J. 1979, Economic Botany of the Guahibo. L Palmae. Econ. Bot. 33 (4): 3611-376.

BALICK M. J. 19811. Jessenia bataua and Oenocarpus especies: native mazonian palms as new sources of edible oil. Pages 145-155 in F. H. Pride, L. H. Princen \& K. D. Mukherjee (eds.), New sources of fats and oils. Am. Oil Chem., Soc.

BALICK M. J. 1982. Palmas neotropicales. Nuevas fuentes de aceite comestible. Interciencia 7 (11): 25-29.

BALICK M. J. 1985. U seful plants of A mazonia: A resource of global importance. Pages 339-367 in G. T. Prance \& T.E. Lovejoy (eds.). Key e environments: A mazonia. Pergamon Press. .

BALICK M. J. 1986. Systematics and economic botany of the Oenocarpus Jessenia (Palmae) complex. Advances in Economic Botany 3:

BALICK M. J. \& SN - GERSHOFF, 1981. Nutritional evaluation of the Jessenia bataua palm. Source of high quality protein and oil from tropical America. Econ. Bot. 25 (3): 261- 271.

BARRAU J. 1959. The sago palms and other food plants of marsh dwellers la the South Pacific islands. Econ. Bot. 13: 151-162.

B00M. B.M. 1986. The Chácobo Indians and their palms. Principes 30: $63-30$

BOUILLENNE R. 1930. Un voyage botanique dans le bas A mazone. Arch. Inst. Bot. U niv. Liége 8: 1-185, pls 1-34 
BRAUN A. 1968. Cultivated palms of V enezuela. Principes 12:39-103, 111-136.

CA VA LCANTE P. B. 1974. Frutas Comestiveis da A mazonia, Publicacoes avulsas 27, M PEG, Belem, Brasil.

FAO-UNESCO, 1971. Soil map of the word, Vol. IV, South A merica. UNESCO, Paris.

FORERO. P. L. E. 1983 A notaciones sobre bibliografía seleccionada del complejo J essenia-O enocarpus (Palmae) Cespedesia 12 (45-46): 21-49

GONZALES, R. M. 1971-74. Estudios sobre la densidad de poblaciones de aguaje (Mauritia sp) en Tingo Maria, Perú. Rev. For. Perú 5 (1-2): 46-54.

GRANVILLE J. J. de, 1987. Recherches sur la flore et la vegation guyanaises. These Doctorat d'E tat, Univ. de M ontpellier.

GRANVILLE J. J. de, 1985. Cueillir sands détruire. SEPANGUY, Cayenne (poster).

GUILLAUMET J. L. 1987. Some structural and floristic aspects of the forest. Experiencia 43: 241-251.

HEINEN, H.D \& K. RUDDLE, 1974. Ecology, ritual and economic organization in the distribution of palm starch among the Warao of the Orinoco delta. Jour Anthropol. Res. 30: 116-138.

KAHN F. 1987 Ecology of economically important palms in Peruvian Amazonia. Advances in Economic Botany 6: 46-53.

KAHN F. \& K. MEJIA 1986. The American oil palm, Elaeis oleifera, Peruvian A mazonia. Principes 30 (4): 182.

KAHN F. \& K. MEJIA, 1987. Notes on the biology, ecology and use a small A mazonian palm: Lepidocaryum tessmanni. Principes 31 (1): 14-19.

KAHN E., MEJIA \& A. de \& CASTRO, 1988. Species richness and density of palms in terra firme forests of amazonia. Biotropica 20. 
LEVI-STRAUSS C. 1950. The use of wild plants in tropical South A merica. Hand book of South A merica Indians 6: 465-486. Cooper Square Publishers, N.Y

LOPEZ PARODI J. 1987. Use of palms in low-cost rural housing in the Peruvian A mazon. Advances in Economic Botany 6. (en prensa)

MARTIN 0. \& Ph. GUICHARD, 1979. A propos de quatre palmiers spontanes d'A mérique latine. Oléagineux 34: 375-381.

MEJIA K, 1983, Palmeras y el Selvícola Amazónico. Univ. Nac. Mayor San $\mathrm{M}$ arcos. M us Hist. N at. Lima.

MEJIA K. 1987 Palm ethnobotany in eleven Mestizo villages in the Peruvian A mazon. Advances in Economic Botany 6. (en prensa)

MEUNIER J. 1976. L es prospection de palmacées. U ne nécessité pour l'amélioration des palmiers oléagineux. Oléagineux 31:153-157.

MOORE H, E. 1973. Palms in the tropical forest ecosystems of Africa and South A merica. Pages 63-88 in B.J. M eggers, E.S. A yensu, \& W.D. Duckworth (eds.), Tropical forest ecosystems in Africa and South America: A comparative review. Smithsonian Institution Press, Washington D. C.

ONERN 1977. U se of remote sensing systems evaluating the potential of the aguaje palm in the Peruvian jungle. ONERN, Lima.

OOI. C., E. B. SILVA, A. A. MULLER, \& J.C. NA SCIM LENTO. 1981. Oil palm genetic resources. Native Elaeis oleifera populations in Brazil offer promising sources. Pesq. A gropec. Bra. Brasilia 16 (3): 385-395.

PADOCH C,, 1987. Marketing of palm products in Iquitos, Peru, with emphasis on A guaje (Mauritia flexuosa). Advances in Economic Botany 6. (en prensa)

PRANCE G.T, 1980. A terminologia dos tipos de florestas amazónicas sujeitas a inundaçáo. A cta A mazonica 10 (3): 499-504.

PROFIZI J. P. 1983. Contribution al étude des palmiers Raphia du Sud Bénin. Botanique. Ecologie. Ethnobotanique Thése Doctorat de spécialité, Univ. of M ontpellier. 
RUDDLE K., D. JOHNSON, P. . TOWNSEND, \& Y,D.REES, 1978. Palm sago. A tropical starch from marginal lands. Univ. of Hawai, Honolulu.

SALAZAR A. \& J. ROESSL, 1977. Estudio de la potencialidad industrial del aguaje. Proyecto ITINTEC n. 3102 UNA-IIA, Lima.

SCHULTES R.E. 1974. Palms and religion in the Northwest A mazon. Principes 18: 3-21.

SCHULTES R. E. 1977. Promising structural fiber palms of the Colombian A mazon. - Principes 21:72-82.

SCHWYZER A. 1981. Producción casera del aceite de ungurahui (Jessenia Polycarpa). Proyecto de Asentamiento Rural Integral-Jenaro Herrera, Iquitos, Perú. Bol. Tec. No 11

SFR UCE R. 181. Palmae A mazonicae, sive Enumeratio Palmarum in itinere suo per regiones A mericae aequatorial es lectarum. J. Linn. Soc. 11:65-183.

WALLACE A. R. ;853 Palm trees of the A mazon and their uses John V an Voorst, London. 